\title{
Supporting Information \\ Dual-stimulus control for ultra-wideband and multidimensional modulation in terahertz metasurfaces comprising graphene and metal halide perovskites
}

Maosheng Yang:@, Tengteng Li®@, Xin Yan ${ }^{\dagger *}$, Lanju Liang ${ }^{\dagger *}$, Haiyun Yao ${ }^{* *}$, Zhaoqing Sun§, Jing $\mathrm{Li}^{\ddagger}$, Jie Li ${ }^{\ddagger}$, Dequan Wei ${ }^{\dagger}$, Meng Wang ${ }^{\dagger}$, Yunxia Ye ${ }^{\ddagger}$, Xiaoxian Song ${ }^{\ddagger}$, Haiting Zhang ${ }^{\ddagger}$ and Jianquan Yao"

Institute of Micro-nano Optoelectronics and Terahertz Technology, and School of Mechanical Engineering, Jiangsu University, Zhenjiang, 212013, China

${ }^{\dagger}$ School of Opto-electronic Engineering, Zao zhuang University, Zao zhuang 277160, China

\College of Precision Instruments and Opto-electronics Engineering, Tianjin University, Tianjin 300072, China

${ }^{\S}$ College of Materials Science and Engineering, Key Laboratory of Advanced Functional Materials of Education Ministry of China, Beijing University of Technology, Beijing 100124, China

E-mail: yx1lj68@126.com; lianglanju123@163.com; haiyun1990yao@163.com

${ }^{\circledR}$ Maosheng Yang and Tengteng Li contributed equally to this work 


\section{Photoconductivity measurements}

The differential transmission $(-\Delta \mathrm{T} / \mathrm{T})$ data of the terahertz pulse are employed to extract the real part of photoconductivity of perovskite and graphene thin film under various pump powers.

The real part of the photoconductivity is given by ${ }^{[1]}$

$$
\Delta \hat{\sigma}_{\text {real }}=\frac{n+1}{Z_{0} d}[-\Delta T / T]\left[\frac{1}{1+\Delta T / T}\right]
$$

which in the limit of $-\Delta T / T<<1$ reduces to

$$
\Delta \hat{\sigma}_{\text {real }}=\frac{n+1}{Z_{0} d}[-\Delta T / T]
$$

$Z_{0}$ is the impedance of the free space and equals $377 \Omega$, d is the thickness of the sample $(d<<\lambda)$, and $\mathrm{n}$ is the refractive index of the substrate in the $\mathrm{THz}$ region. The change in terahertz amplitude is defined as $\Delta \mathrm{T}=\mathrm{T}_{\text {pump }}-\mathrm{T}_{\text {nopump. }} \mathrm{T}$ is terahertz transmission through film at no optical pump. ${ }^{[2]}$ Upon different pump fluences, the real part photoconductivity of perovskite and graphene thin film were recorded at the peak $\mathrm{THz}$ amplitude, as shown in Figure S1. With the increase of pump fluences, both the real part photoconductivity of perovskite and graphene thin film enlarge. Figure S1a exhibits the real part of the photoconductivity for different pump fluences. The conductivity values at the peak $\mathrm{THz}$ amplitude were used in the simulation. Modulation of transmission resonance was solely caused by modification of the conductivity in the perovskite and graphene. 

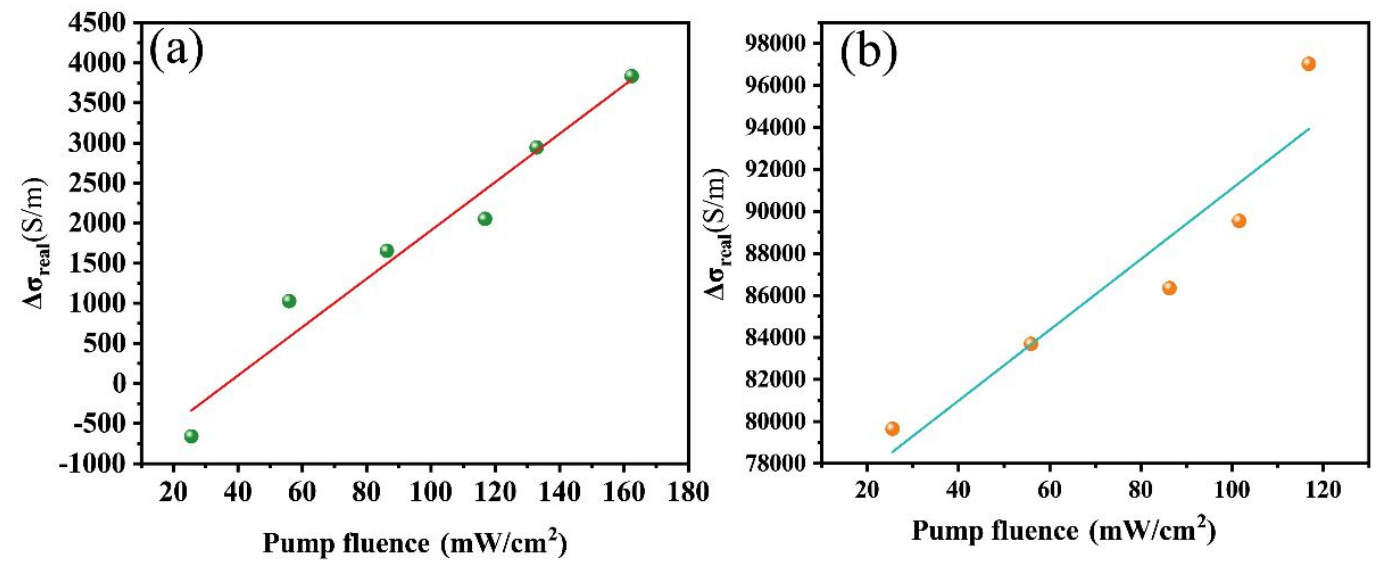

Figure S1 the real photoconductivity at different pump fluences. a) The real photoconductivity of perovskite. b) The real photoconductivity of graphene.

\section{Simulated distributions of the electric field}

We use the asymmetric U-shape structure with the perovskite film alone (without graphene) as an example to explain the modulation mechanism. As illustrated in Figure S1a, the field concentrations in the periphery of the asymmetric U-shape structure are large under the $0 \mathrm{~mW} / \mathrm{cm}^{2}$. It is observed from Figure S1b that upon pump fluences of $55.9 \mathrm{~mW} / \mathrm{cm}^{2}$, the electric fields in the periphery of the asymmetric U-shape structure largely reduced, resulting in the weakening of the $\mathrm{O}$ Fano resonance. Such a trend is further enhanced upon application of $116.8 \mathrm{~mW} / \mathrm{cm}^{2}$ optical pump as shown in Figure S1c, due to the increase in perovskite film conductivity.
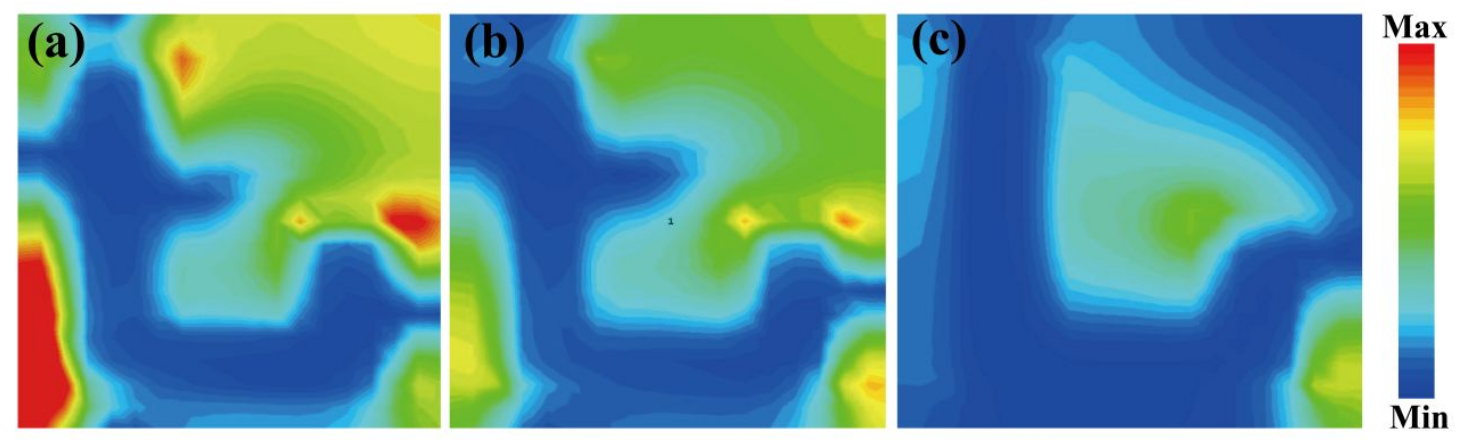

Figure S2 Simulated distributions of the electric field. a-c) absolute electric field of O Fano resonance under 0 to $116.8 \mathrm{~mW} / \mathrm{cm}^{2}$.

\section{The experimental photoelectric equipment and THz-TDS device}

Figure S3 shows the experimental photoelectric equipment and THz-TDS device. The experimental photoelectric equipment contains a 532-nm laser served as an optical pump with a spot size (diameter) of $\sim 2 \mathrm{~mm}$, and a probe system with the function of applying a bias voltage. The THzTDS device is an $8 \mathrm{f}$ confocal terahertz time-domain spectroscopy system. The THz time scan of 40 ps was used to obtain a spectral resolution of $40 \mathrm{GHz}$. 


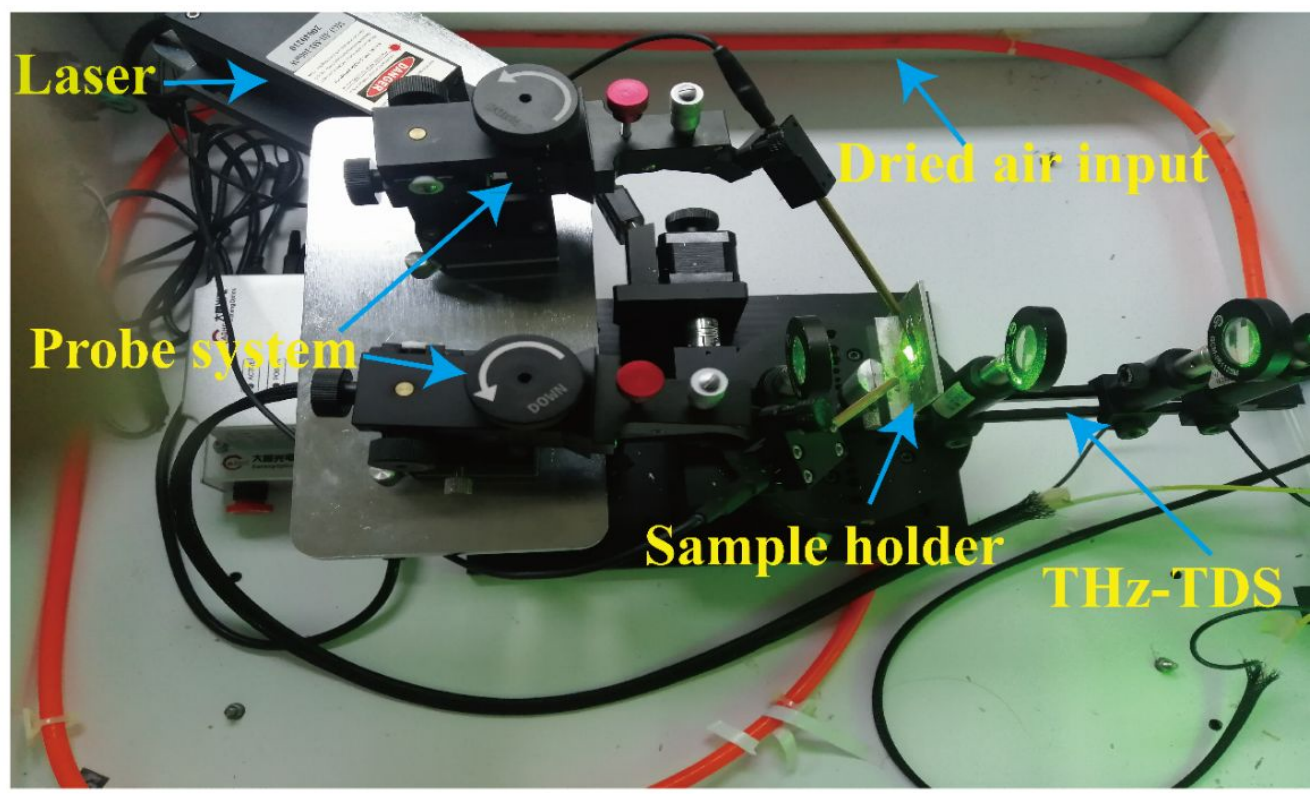

Figure S3 Experimental photoelectric equipment and THz-TDS device

\section{Supporting References}

[1] Jepsen P U, Cooke D, Koch M. Erratum to Terahertz Spectroscopy and Imaging-Modern Techniques and Applications [J]. Laser \& Photonics Reviews, 2012, 6(3): 418.

[2] Kumar A, Solanki A, Manjappa M, et al. Excitons in 2D Perovskites for Ultrafast Terahertz Photonic Devices [J]. Science advances, 2020, 6(8): eaax8821. 